\title{
Laser-controlled Injector for Biological Applications
}

\author{
Toshihiko OOIE $^{* 1}$, Masato TANAKA ${ }^{* 1}$, Tomonori NAKAHARA $^{* 1}$, Yuji YAMACHOSH ${ }^{* 1}$, \\ Rie AKAMINE ${ }^{* 1}$, Masatoshi KATAOKA ${ }^{* 1}$, Yoshinobu BABA ${ }^{* 1 * 2}$ \\ ${ }^{* 1}$ Health Research Institute, AIST, \\ Shikoku Center, 2217-14 Hayashi-cho Takamatsu, Kagawa 761-0395, Japan \\ E-mail: toshihiko-ooie@aist.go.jp \\ ${ }^{* 2}$ Graduate School of Engineering, Nagoya University, Furo-cho, Chikusa-ku, \\ Nagoya 464-8603, Japan
}

\begin{abstract}
A novel injector for biological applications has been developed. It is a drop-on-demand injector driven by a pulsed laser. Explosive vapor bubbles formed on a thin metal foil, which was irradiated by laser beam, effectively ejected pure water or the biopolymer samples in water solution from a microchannel as small droplets through the port. The size and flight speed of ejected droplets were measured by CCD camera equipped with microscope and synchronized strobe light. The heat efficiency of the injector was evaluated to compare with that of conventional thermal inkjet unit. Furthermore, antigen-antibody reaction using ejected antibody solution was tested. Ejected antibody dots for procollagen type I c-peptide (P1CP) were successfully immobilized on a cyclo olefin copolymer (COC) substrate with a commercially available polymer coating. By using formed antibody dots for a sandwich ELISA the P1CP antigen was effectively detected showing that the injector is applicable for biological application such as micro immunoassay chip.
\end{abstract}

DOI:10.2961/jlmn.2011.01.0010

Keywords: fiber laser, droplet, microfluidic, inkjet, COC, biomarker, antibody, antigen

\section{Introduction}

Enzyme-linked immunosorbent assay (ELISA) has been used commonly as a diagnostic tool in blood test. ELISA method requires a sample volume of $20 \mu \mathrm{l} /$ item or more and at least three hours for antigen-antibody reaction. In order to reduce the sample volume and shorten the reaction time, we developed a chip that allowed for the antigen-antibody reaction on a microscopic region in a microchannel. The chip also allowed multiple immunoassays in a channel. In the development, we used an injector driven by a piezoelectric actuator. For further applications of the chip, such as personalized healthcare and the evaluation of an antibody, a disposable injector with small or no buffer volume is necessary.

J. Deoksuk et. al. experimentally analyzed the bubble dynamics of ink, the absorption coefficient of which was sufficient for direct heating, induced by laser irradiation [1]. Their results show that the bubble generated in the ink by a laser pulse is similar to that produced by an electric thin film heater.

Indirect laser heating via metal film was applied for the phosphor layer deposition to a plasma display panel (PDP) by J. H. Lee et. al. [2]. However, their process is a transfer rather than an inkjet, since they used a shallow, hemispherical, blind hole without any supply line to refill liquid.

Transfer techniques involving laser-induced forward transfer (LIFT) [3] have been studied for biological applications [4-8] also. Various biological materials including proteins, DNA and cells have been transferred without significant damage.

We have developed an injector unit driven by a pulsed laser. By using external laser unit and the pressure of vaporized liquid in a micro channel, this injector unit requires only a micro channel, a piece of metal foil as a laser absorber plus an ejection port on the chip.

\section{Experimental}

In the experiments of the laser controlled injector (LIJ), a fiber laser (wavelength 1060nm, YLD-40, IPGPhotonics) was used to heat a thin metal foil, which plays the role of a heater in thermal inkjet process.

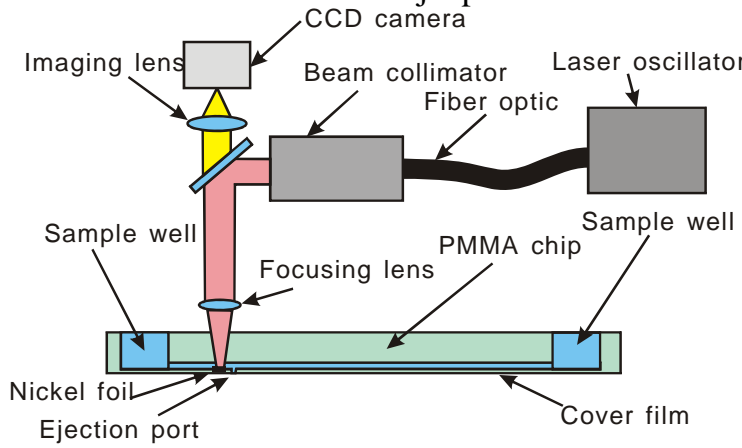

Fig.1 Schematic diagram of a laser controlled injector.

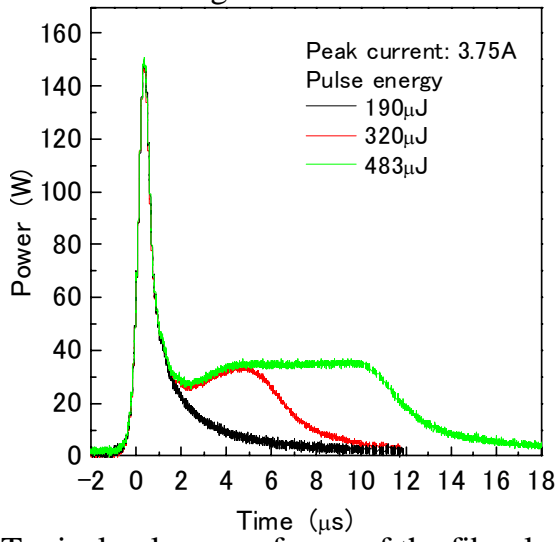

Fig.2 Typical pulse waveforms of the fiber laser used for LIJ. 
The laser beam was focused by a lens, the focal length of which was $40 \mathrm{~mm}$, onto the foil (Fig.1). The focus was set at $300 \mu \mathrm{m}$ below the absorber surface creating a beam diameter of approximately $75 \mu \mathrm{m}$. As shown in Fig.2, the pulse consisted of two parts, an initial high, narrow peak full width at half maximum of which was constant at 800ns and tail that depends on the pulse width.

\subsection{Microfabrication of LIJ}

Our injectors were made by using 4 laser, 1 etching and 2 manual processes as follows (Fig.3).

(1) Polymethilmethacrylate (PMMA) plates (CLAREX ${ }_{\circledast}$, Nitto Jushi Kogyo Co., Ltd.) were used for base material. The shape line and the two sample inlet ports are cut by a $\mathrm{CO}_{2}$ laser.

(2) Micro grooves connecting two inlet ports are formed by an ArF excimer laser, of which pulse energy, maximum power, maximum repetition rate and pulse width were $120 \mathrm{~mJ}, 20 \mathrm{~W}, 200 \mathrm{pps}$ and $14 \mathrm{~ns}$, respectively. The magnification of the optics was 1/10 and the mask was oblong with aperture of $1 \times 10 \mathrm{~mm}$. The fluence at the processing surface calculated from the beam energy after the object lens and the area of irradiation is $3 \mathrm{~J} / \mathrm{cm}^{2}$. Laser repetition rate was fixed at $5 \mathrm{~Hz}$ in this process. The width and the depth of the groove were $100 \mu \mathrm{m}$ and $50 \mu \mathrm{m}$, respectively.

(3) The debris and the laser affected layer on the groove surface are removed by GG solution etching for $1 \mathrm{~min}$ in an ultrasonic bath [9]. The organic solvent mixture is known as "GG Developer (U.S. Patent No. 4393129)".

(4) Thin nickel foils are manually set on the grooves. The grooves are covered by a film (PMMA film with acrylate adhesive layer, Toyo Ink Mfg. CO., Ltd) to form micro channels. The size and the thickness of the foils were $150 \times 150 \mu \mathrm{m}$ and $2.5 \mu \mathrm{m}$, respectively. To minimize the heat affection, ultrafast laser was used to cut the foils.

(5) The cover film is drilled by the ArF excimer laser described in (2) with a circular mask to form ejection ports.

(6) The injectors are cut off manually.

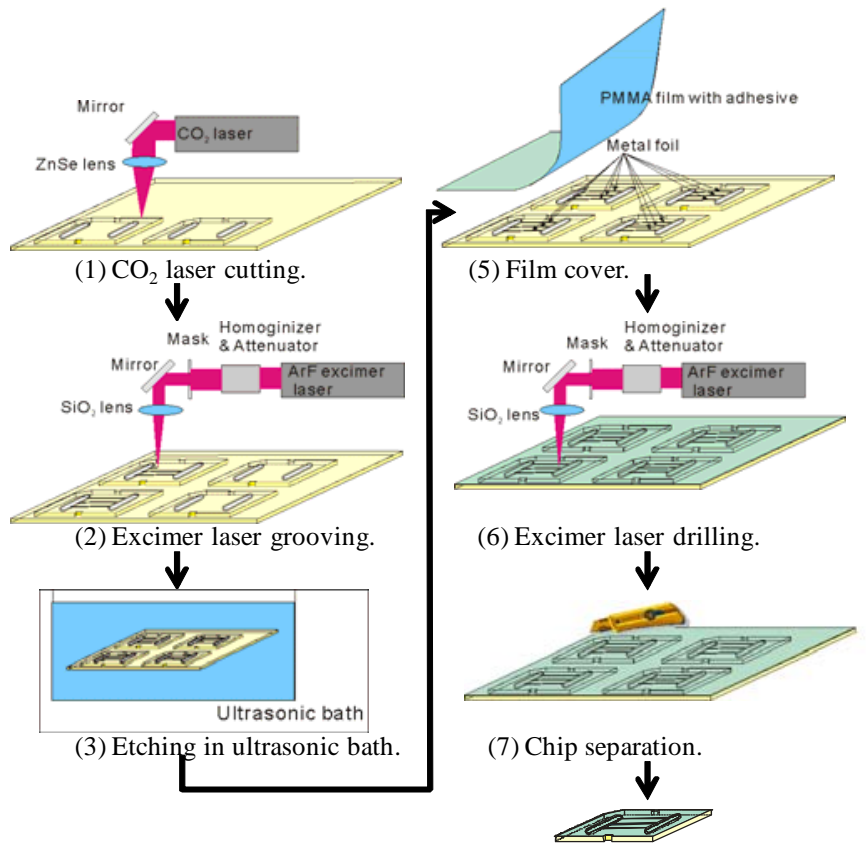

Fig.3 Microfabrication for experimental LIJ.

\subsection{Observation of ejected droplets}

A CCD camera equipped with a microscope and a strobe light synchronized with certain delay time to the laser pulse was used to record the images of ejected droplets in order to measure the size and the flight speed (Fig.4).

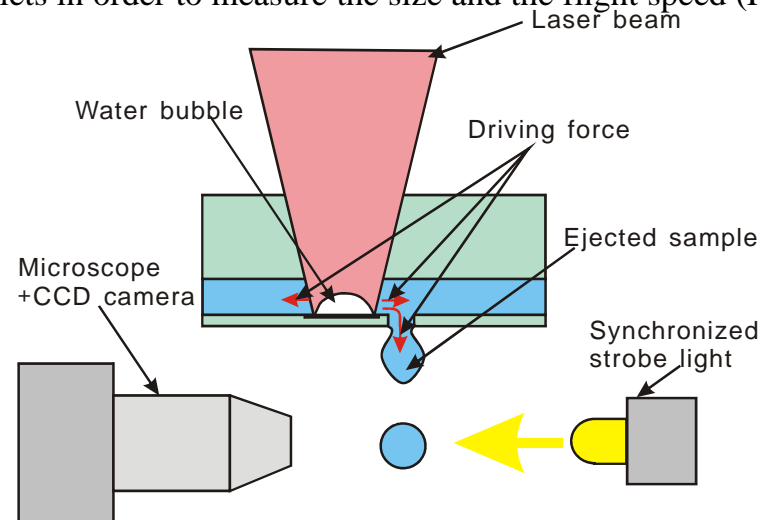

Fig.4 Imaging of ejected droplets using a CCD camera equipped with synchronized strobe light.

\subsection{P1CP antigen detection}

The antigen detection tests with the antibody dot formed by LIJ and an injector driven by a piezoelectric actuator (PIJ) were operated as follows, respectively.

(1) Ejection of procollagen type I c-peptide (P1CP) antibody solutions by LIJ and PIJ onto the surface of a cyclo olefin copolymer (COC) substrate with a polymer coating.

(2) Leave the substrate at room temperature for four hours.

(3) Wash in a sealed case filled with cleaning solution.

(4) Drop the compound liquid containing the P1CP antigen, the concentration of which was $640 \mathrm{ng} / \mathrm{ml}$, and the secondary antibody labeled with a peroxidase on the immobilized antibody.

(7) Leave for 30min.

(8) Wash in a sealed case filled with cleaning solution.

(9) Drop a chemiluminescence substrate.

(10) Measure the luminescence signal with a cooled CCD camera.

\section{Results and discussion}

The experimental injector shown in Fig.5 was composed of an absorber, a film with ejection port and a laser grooved PMMA plate. The port size at outer surface is larger than that at inner surface.

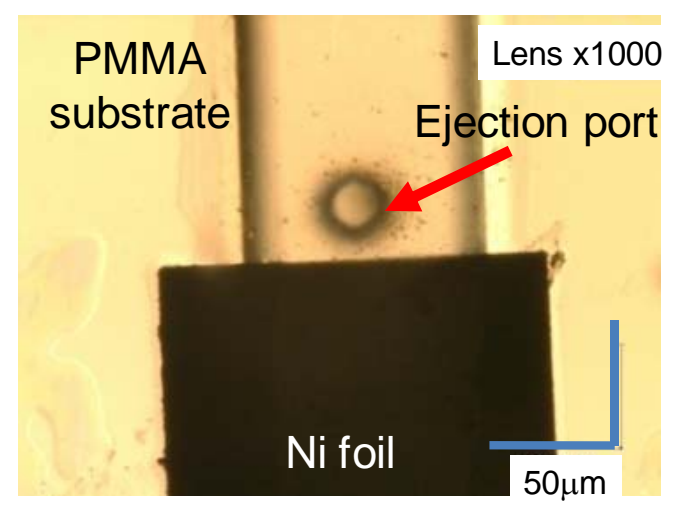

Fig.5 Photograph of an experimental injector for LIJ. 


\subsection{Ejection performance of LIJ}

The images of ejected droplets at various time delays after the rise of the laser pulse are shown in Fig.6. The distance from the ejection port to the droplet is proportional to the delay time up to $400 \mathrm{~ms}$, showing that the flight speed is constant in that region.

The flight speed of the droplets slightly depends on the peak power of the laser pulse under constant pulse energy as shown in Fig.7. However, it strongly depends on the pulse energy and the diameter of the ejection port under the constant peak power as shown in Fig.8.

B. Hockwind et.al. [10] configured the energy effi-

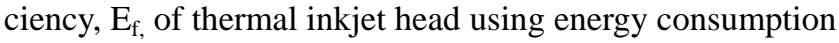
by heater, $E_{h}$ and the energy of ejected droplet, $E_{d}$, as:

$$
E_{F}=\frac{E_{d}}{E_{h}}
$$

The energy of ejected droplet is obtained by the kinetic energy, $E_{k}$, and the surface energy, $E_{s}$, as:

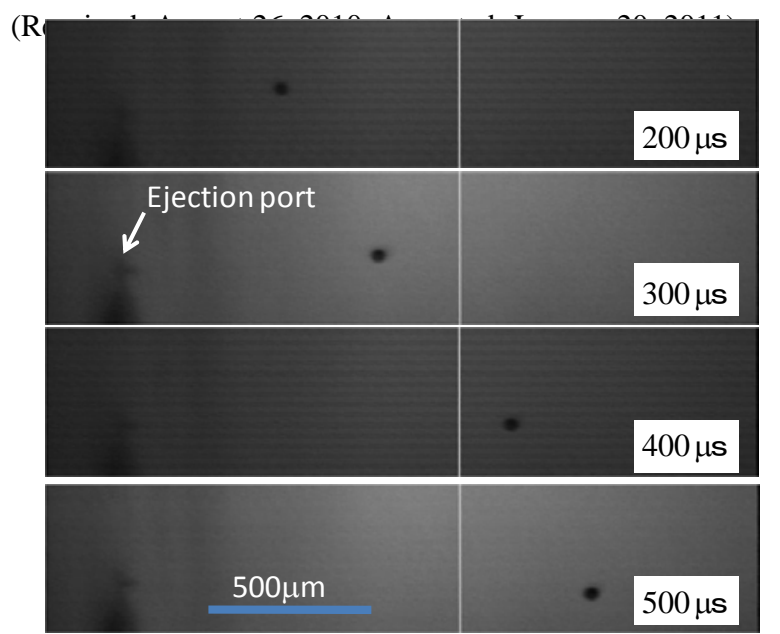

Fig.6 Droplet images at various time delays after ejection.

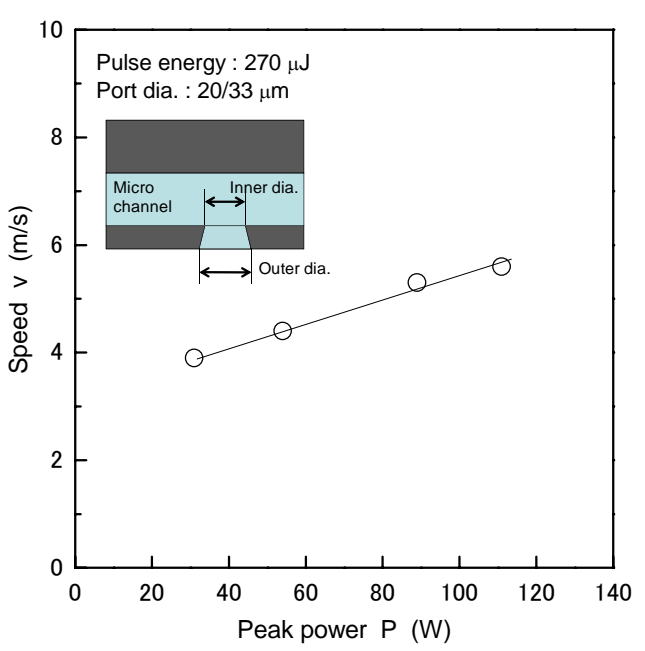

Fig.7 Relationship between the speed of ejected droplets and laser power at the peak of pulses.

$$
E_{d}=E_{k}+E_{s}=\frac{1}{2} \cdot m_{d} \cdot v_{d}^{2}+4 \cdot \pi \cdot r_{d}^{2} \cdot \sigma
$$

where $m_{d}, v_{d}, r_{d}$ and $\sigma$ are the mass, the speed, the radius and the surface tension of ejected droplet, respectively.

Using the absorption ratio of laser, A, the energy of laser pulse, $\mathrm{E}_{1}$ and the energy for the temperature rise of absorber, $E_{10}$, we assumed $E_{h}$ of our injector as:

$$
E_{h}=A \cdot\left(E_{l}-E_{l 0}\right)
$$

By Eq. (1), (2) and (3), $v_{d}$ is expressed as follows:

$$
v_{d}=\sqrt{\frac{2 \cdot E_{F} \cdot A\left(E_{l}-E_{l 0}\right)-8 \cdot \pi \cdot r_{d}{ }^{2} \cdot \sigma}{m_{d}}}
$$

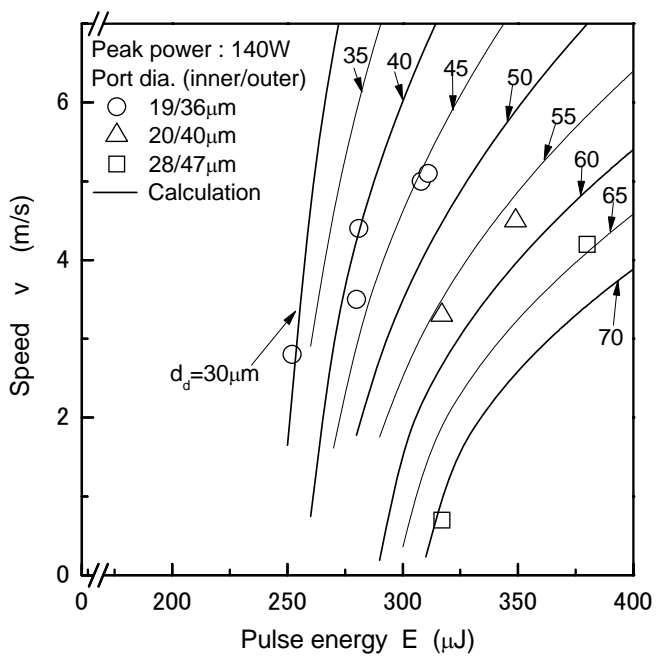

Fig.8 Relationship between the flight speed of ejected droplets and laser energy. Solid lines indicate the results of calculation at various diameters of droplets, $\mathrm{d}_{\mathrm{d}}$.

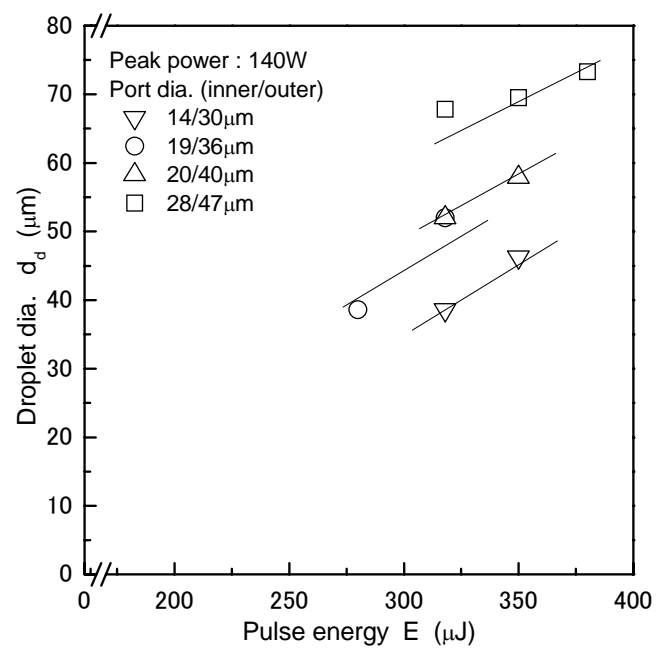

Fig.9 Effect of pulse energy on droplet diameter $d_{d}$. 
We used Eq. (4) to compare the energy efficiency $E_{F}$ of LIJ to conventional thermal inkjet head. $\mathrm{E}_{\mathrm{F}}$ and $\mathrm{E}_{\mathrm{l} 0}$ in Eq. (4) are head parameters. Data points and solid lines in Fig.8 show measured and calculated flight speeds of droplets, respectively. Following $E_{F}$ and $E_{10}$ were used for calculation.

$$
\begin{aligned}
& E_{F}=5 \times 10^{-5} \\
& E_{l 0}=235 \mu \mathrm{J}
\end{aligned}
$$

The laser absorption ratio of nickel at $1.06 \mu \mathrm{m}(\mathrm{A}=0.3)$ and the surface tension of pure water $(\sigma=72.75 \mathrm{mN} / \mathrm{m})$ were used as A and $\sigma$, respectively. In Fig.8, by a calculated line that goes across the data point, the diameter of the droplet for each data point is obtained. As shown in Fig.9, we also measured the diameters of droplets experimentally at various pulse energies using magnified images of droplets recorded by same system as Fig.4. According to Fig.8, the diameters of droplets in $19 / 36 \mu \mathrm{m}, 20 / 40 \mu \mathrm{m}$ and $28 / 47 \mu \mathrm{m}$ ports are $30-50 \mu \mathrm{m}, 55-60 \mu \mathrm{m}$ and $65-70 \mu \mathrm{m}$, respectively. These values and their tendency to increase with increasing pulse energy agree well to measured droplet sizes shown in Fig.9 showing that similar model to thermal inkjet head is applicable for our LIJ head.

The $\mathrm{E}_{\mathrm{F}}, 5.0 \times 10^{-5}$, is close to the reported value in a thermal inkjet head with side-shooter geometry [11], the range of which was $8-15 \times 10^{-5}$. Since the energy efficiency depends on the geometry of the injectors in general, it is not easy to improve $E_{F}$ keeping the simple geometry. On the other hand, $\mathrm{E}_{10}$ value is close to the energy to increase the temperature of the absorber by $300 \mathrm{~K}$, where the ejection starts, showing that the decrease of absorber thickness would dramatically decrease the laser pulse energy for the injector.

\subsection{Antigen detection by LIJ immobilized antibody}

Procollagen type I c-peptide solution dots on a COC substrate ejected by LIJ, (a) and (b), and PIJ, (c) and (d) are shown in Fig.10. The volume of the ejected solution by LIJ and PIJ were 45pl and 113pl, respectively. Since only PIJ has a water repelling coat around the ejection port, the directivity of PIJ is superior to that of LIJ, however, the directivity of LIJ is fair for the ejection to the bottom of microgroove of our single channel multiple immunoassay chip, the width and the depth of which are $300 \mu \mathrm{m}$ and $100 \mu \mathrm{m}$, respectively [12].

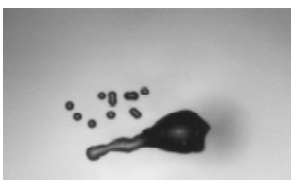

(a) LIJ, 50drops

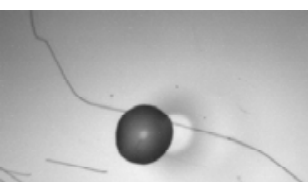

(c) PIJ, 50drops

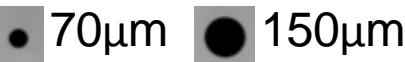

Fig.10 Photographs of P1CP antibody solution ejected on a COC substrate at $1 \mathrm{~mm}$ from the ejection port.
Fig.11 shows the results of P1CP antigen detection by the antibody dots immobilized by LIJ and PIJ. In our previous report, the single-channel multiple immunoassay chip with PIJ immobilized antigen dots indicated superior sensitivity and speed to conventional ELISA [12]. The geometry of the chip is different from the previous one, however, the antibody dots immobilized by LIJ indicates similar sensitivity to that by PIJ showing that the deterioration of antibody by LIJ ejection is not significant, similar level to PIJ at least. A disposable LIJ would be a powerful tool for biological applications and on-demand manufacturing of a tailor-made micro-channel chip.

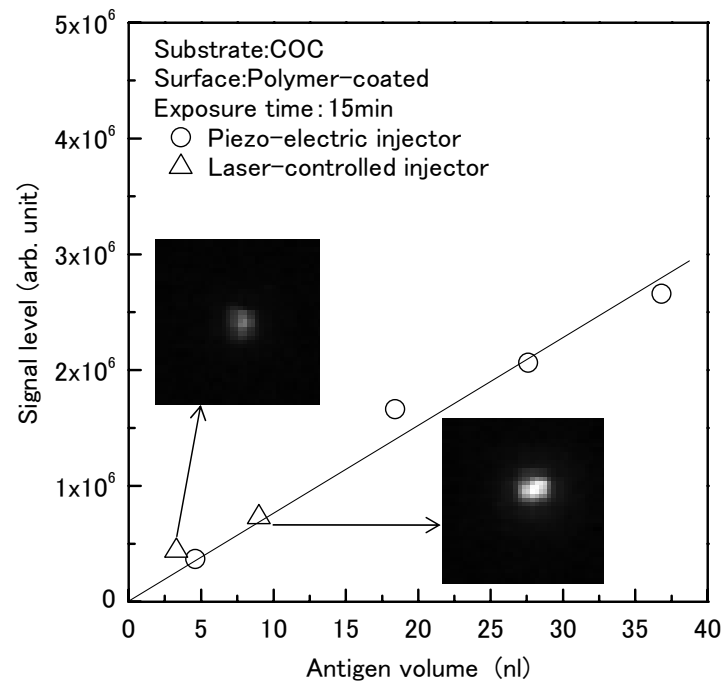

Fig.11 Results of antibody detection tests. The concentration of P1CP antibody solution ejected by the injectors and the concentration of P1CP antigen tested were $0.1 \mathrm{mg} / \mathrm{ml}$ and $640 \mathrm{ng} / \mathrm{ml}$, respectively.

\section{Conclusions}

A novel injector for biological applications has been developed. In LIJ, the flight speed of ejected droplets depends on the pulse energy of the laser rather than the peak power. A simple energy consumption model fit well to the experimental results. Estimated heat efficiency of LIJ was $5 \times 10^{-5}$, close to the value of a thermal inkjet head. The laser energy would dramatically decrease by reducing the thickness of laser absorber. Furthermore, we have succeeded to detect P1CP antigen by the antibody dots ejected by PIJ, showing that the deterioration in antibody-antigen reactivity by LIJ ejection is not significant in P1CP antibody.

\section{References}

[1] J. Deoksuk, J. Hong, K. Dongsik, C. Sung-Hoon: J. Imaging Sci. Technol., 50 (2006)173.

[2] J. H. Lee, C. D. Yoo and YS. Kim: J. Micromech Microeng, 17 (2007)258.

[3] D. A. Willis and V. Grosu, Applied Physics Lett.: 86 (2005)244103.

[4] P. Serra, M. Colina and J. M. Fernandez-Pradas: Applied Physics Lett., 85 (2004)1639.

[5] B. R. Ringeisen, H. Kim, J. A. Barron, D. B. Krizman, D. B. Chrisey, S. Jackman, R. Y. C. Auyeung, B. J. Spargo: Tissue Engineering, 10 (2004) 483. 
[6] M. Colina, M. Duocastella, J. M. Fernandez-Pradas, P. Serra and J. L. Morenza: J. Applied Physics, 99 (2006) 084909

[7] P. Serra, J. M. Fernandez-Pradas, M. Colina, M. Duocastella, J. Dominguez and J. L. Morenza: Journal of Laser Micro/Nanoengineering, 1 (2006) 236.

[8] M. Duocastella, J. M. Fernandez-Pradas, P. Serra, J. L. Morenza: Proc. LPM2007, Viena (Austria), (2007), on line.

[9] H. Miyashita, T. Ooie, T. Nakahara, Y. Baba: Journal of Laser Micro/Nanoengineering, 3 (2008) 88.

[10] B.Hockwind: Proc. IS\&T's $9^{\text {th }}$ International Congress on Advances in Non-Impact Printing Technologies, Yokohama, (1993) 237.

[11] M. Fujii: The Journal of Imaging Science and Technology, 43 (1999) 332.

[12] M. Tanaka, T. Ooie, Y. Yamachoshi, T. Nakahara, M. Hino, R. Akamine, M. Kataoka: Journal of Laser Micro/Nanoengineering, 5 (2010) 35.

(Received: August 26, 2010, Accepted: January 20, 2011) 